\title{
Effects of three different training modalities on the cross sectional area of the lumbar multifidus muscle in patients with chronic low back pain
}

\author{
L A Danneels, G G Vanderstraeten, D C Cambier, E E Witvrouw, J Bourgois, \\ W Dankaerts, H J De Cuyper
}

\begin{abstract}
Objectives-To determine the effect of different training schedules on the cross sectional area (CSA) of the lumbar multifidus muscle in patients with chronic low back pain.

Methods-Each of 59 nine patients was randomly assigned to one of three programmes: 10 weeks of stabilisation training (group $1 ; n=19$ ); 10 weeks of stabilisation training combined with dynamic resistance training (group $2 ; \mathbf{n}=$ 20); 10 weeks of stabilisation training combined with dynamic-static resistance training (group $3 ; n=20$ ). Before and after 10 weeks of training, multifidus CSAs were measured from standard computed tomography images at three different levels (upper end plate of L3 and L4, and lower end plate of L4).

Results-The CSA of the multifidus muscle was significantly increased at all levels after training in group 3 . In contrast, no significant differences were found in groups 1 and 2.

Conclusions-General stabilisation exercises and dynamic intensive lumbar resistance training have no significant effect on the CSA of the lumbar multifidus muscle in patients with chronic low back pain. The static holding component between the concentric and eccentric phase was found to be critical in inducing muscle hypertrophy during the first 10 weeks. Treatment consisting of stabilisation training combined with an intensive lumbar dynamicstatic strengthening programme seems to be the most appropriate method of restoring the size of the multifidus muscle.

(Br F Sports Med 2001;35:186-191)
\end{abstract}

Keywords: back pain; multifidus muscle; stabilisation; dynamic; dynamic-static; hypertrophy

Department of Physical Medicine and Rehabilitation, Hospital Jan Palfijn-Campus Gallifort, Antwerp, Belgium

H J De Cuyper

Correspondence to: Mr Danneels, University Hospital Gent, Department of Rehabilitation Sciences and Physical Therapy, De Pintelaan 185, 1B3, B-9000 Ghent, Belgium

lieven.danneels@rug.ac.be

Accepted 18 January 2001 weakness. Biomechanical and clinical studies have shown that muscles can provide segmental stabilisation by controlling motion in the neutral zone, and the neutral zone can be regained to within physiological limits by effective muscle control. ${ }^{9}$

Many authors have highlighted the importance of the lumbar multifidus muscle in providing dynamic control. ${ }^{8} 10^{11} 12$ In a recent computed tomography (CT) study, we found selective atrophy of the multifidus muscle in $\mathrm{CLBP},{ }^{13}$ and, in a magnetic resonance image (MRI) study by Kader et al, ${ }^{14}$ multifidus muscle atrophy was present in $80 \%$ of patients with low back pain. This may permit spinal instability and therefore be an important factor in the high rate of recurrence of CLBP.

Objective direct measurement of muscles may help in the assessment of low back pain and aid the choice of appropriate treatment. ${ }^{15}$ Morphological information on muscles can be obtained in a non-invasive way by $\mathrm{CT}^{13}{ }^{16-19}$ and MRI. ${ }^{14}{ }^{21-23}$ However, despite cumulative evidence that the cross sectional area (CSA) of the paraspinal muscles is smaller in patients with $\mathrm{CLBP}^{132124}$ and postoperative low back pain, ${ }^{319}$ this variable is rarely used to evaluate treatment programmes.

There is general consensus in the literature supporting the need for active reconditioning exercise in the treatment of CLBP. ${ }^{25}$ Unfortunately, there is little agreement on which exercise regimens are most effective. Information on the effects of different contraction modalities is lacking. However, the type of muscle work seems to be important.

The use of static stabilisation training has been advocated by Jull and Richardson ${ }^{26}$ as an ideal means of improving the recruitment of back muscles capable of enhancing spinal stability, particularly the multifidus. On the other hand, others support the use of high loaded dynamic exercises for successful management of back pain. ${ }^{27}$ Eccentric muscle contractions seem to be essential to obtain optimal hypertrophy in response to resistance training, ${ }^{28}$ and a combined dynamic-static training mode has been recommended in order to recruit as many motor units as possible. ${ }^{30}$ This training method is often used in sports management and muscle rehabilitation, but, to our knowledge, its effect on the CSA of back muscles in patients with CLBP has not been studied.

The objective of this study was to determine whether multifidus muscle atrophy can be reversed. Therefore the volume increasing effect of three different treatments on the multifidus is analysed. 


\section{Materials and methods SUBJECTS}

After approval had been obtained from the ethics committee of the Hospital Jan Palfijn, Antwerp, 59 patients with CLBP were enrolled in the study. All had suffered from back pain for more than three months. A full medical screening examination was carried out, which included a lumbar $x$ ray and neurological examination. Exclusion criteria were previous lumbar surgery, spinal abnormalities indicated on radiographs (the presence of spondylolysis or spondylolisthesis, a lumbar scoliosis exceeding $10^{\circ}$ ), neuromuscular or joint disease, reflex and/or motor signs of nerve root compression or cauda equina compression, evidence of systemic disease, carcinoma, or organ disease, and pregnancy. Patients carrying out sports or fitness training for the low back muscles over the preceding three months were excluded.

\section{STUDY DESIGN}

A randomised clinical trial, test-retest design was used. At entry to the trial, patients signed an informed consent form. In all subjects, standardised transaxial CT images were produced at three levels by an independent radiologist blind to the content of the study. During the recruitment period, each patient was randomly assigned to one of three rehabilitation programmes: stabilisation training (group $1 ; n=19$ ) or stabilisation combined with dynamic (group $2 ; \mathrm{n}=20$ ) or dynamic-static resistance training (group 3; $\mathrm{n}=20$ ). The intervention period was 10 weeks. At the completion of the intervention, new CT images were produced by the same radiologist. Table 1 gives the group characteristics at entry to the trial.

\section{TREATMENT}

The intervention period was 10 weeks at a frequency of three times a week. Each session started with 10 minutes application of warmth (diathermy), followed by active exercises and massage of the lumbar region. In group 1, the active exercises consisted of stabilisation training, and in groups 2 and 3 the same stabilisation training was combined with an intensive lumbar strengthening programme for the back extensors.

\section{Group 1}

The stabilisation training was based on a set of daily living activities in a variety of starting positions and was intended to activate the multifidus in a specific progression of exercises as

Table 1 Characteristics, at entry to the trial, of patients with low back pain grouped according to the training programme

\begin{tabular}{lcccc}
\hline Variable & $\begin{array}{l}\text { Group 1 } \\
(n=19)\end{array}$ & $\begin{array}{l}\text { Group 2 } \\
(n=20)\end{array}$ & $\begin{array}{l}\text { Group 3 } \\
(n=20)\end{array}$ & p Value \\
\hline Age (years) & $43(13)$ & $44(12)$ & $43(12)$ & 0.72 \\
Height $(\mathrm{cm})$ & $170(10)$ & $169(11)$ & $171(9)$ & 0.95 \\
Weight (kg) & $71(14)$ & $70(14)$ & $70(13)$ & 0.78 \\
CSA of multifidus & & & & \\
$\quad$ L3 upper & $0.341(0.058)$ & $0.385(0.078)$ & $0.348(0.097)$ & 0.19 \\
L4 upper & $0.547(0.139)$ & $0.519(0.096)$ & $0.498(0.098)$ & 0.50 \\
L4 lower & $0.71(0.161)$ & $0.725(0.137)$ & $0.635(0.126)$ & 0.145 \\
\hline
\end{tabular}

Values are mean (SD). The cross sectional area (CSA) of the multifidus muscle is expressed as a percentage of the CSA of the vertebral body. Group 1, 10 weeks of stabilisation training; group 2, 10 weeks of stabilisation training combined with dynamic resistance training; group 3, 10 weeks of stabilisation training combined with dynamic-static resistance training. described by O'Sullivan et al. ${ }^{31}$ The patients were asked to keep a physiological lordosis of the lumbar spine while performing the exercises. The aim was to enhance the dynamic stability of the lumbar spine in a functional manner. During this type of exercise, the magnitude of resistance for the trunk muscles is at about $30 \%$ of their maximum in line with their holding and controlling role. ${ }^{26}$

\section{Groups 2 and 3}

In group 2 (dynamic) and group 3 (dynamicstatic), the stabilisation training was combined with progressive resistance training. This training consisted of three standard exercises. The first exercise consisted of leg extension in the four point kneeling position. The subject was positioned on the floor with both knees, hips, and shoulders flexed at $90^{\circ}$, and one leg was extended to $0^{\circ}$ in the knee and the hip, and then returned to the starting position; the exercise was repeated with the contralateral leg. The second exercise was trunk lifting from a prone position on a couch with strap fixation over the calves. With hands placed on the forehead, the trunk was lifted to the greatest possible extension in hips and spine. The third exercise consisted of leg lifting from a prone position on a couch. Patients stabilised themselves by grasping the rim of the couch. Both legs were lifted to the greatest possible extension in hips and spine. For the progressive resistance training, the intensity was objectively quantified by controlling exercise intensity (percentage of repetition maximum), volume (sets of repetitions), and frequency. In each training session, subjects were required to perform three sets of each exercise. The weight lifted was based on the maximum number of repetitions performed before fatigue prevented completion of an additional repetition. This is referred to as the repetition maximum (RM), and generally reflects the intensity of the exercise. ${ }^{5}$ Usually the weight used is a percentage of the maximum that can be lifted once, generally referred to as a one repetition maximum (1 RM). In this study all patients trained at $70 \%$ of the 1 RM. This allowed 15-18 repetitions until muscular fatigue. When the patients performed less than 15 or more than 18 repetitions, a retest procedure was carried out with an adjusted weight.

When the trunk (exercise 2) or the legs (exercise 3) were too heavy, assistance was provided by weights attached to a cable-pulley system and connected to a belt around the breast (exercise 2) or feet (exercise 3) of the patient. When the patient could perform more than 18 repetitions without assistance, resistance was given by a weight fixed around the ankles (exercises 1 and 3 ) or by a weight held in front of the neck (exercise 2).

Each repetition was performed in a standardised and controlled manner (metronome at 60 beats $/ \mathrm{min}$ ), allowing two seconds for the concentric movement and two seconds for the eccentric movement. For group 2, the concentric and eccentric movements were alternated. For group 3, this cycling movement was interrupted each time by a five second static 
contraction between the concentric and eccentric phase.

COMPUTED TOMOGRAPHY

Macroscopically, the size of muscles can be studied by CT and MRI. MRI is preferred because it is a non-ionising radiation technique. However, because of the availability of the different devices and the experience acquired in previous research, CSA was measured in this study by CT. The procedure in all subjects included three standardised transaxial images, positioned accurately through an end plate of a vertebral body, the first through the upper end plate of L3, the second and third through the upper and lower end plate of L4 (fig 1). Some authors have reported that the CSA of the paraspinal muscles is maximal or near maximal at the upper end plate of L4, ${ }^{162132}$ and others have found that the total CSA of the back muscles is maximal at about the $\mathrm{L}^{17}{ }^{33}$ or L4-L5 level. ${ }^{18} 1922{ }^{23}$ In this study, in order to screen the most suitable level and to detect a possible systematic difference between the different levels, three levels were analysed. Instead of the upper end plate of L5, the lower end plate of L4 was chosen because in many people the L5 vertebra is steeply angulated. ${ }^{18}$ Because of such an angulation, the sections would not be comparable with the other levels.

Joint position and muscle length influence CSA values of the muscles, therefore every attempt was made to standardise the position. During CT, each subject was positioned in a prone position with hips in a neutral position to avoid compression of the back muscles. Lumbar lordosis was minimised by placing a pillow under the abdomen. The subjects were instructed to relax and stay motionless for the duration of the scan. A physiotherapist, trained in this method, positioned the patients and controlled the back musculature by palpation to ensure a relaxed state.

A CT Pace Plus (General Electric) was used at $120 \mathrm{kV}$ and $160 \mathrm{~mA}$. A window width of 400 Hounsfield units (HU), with the centre at +4 $\mathrm{HU}$, was used. The slice thickness was $5 \mathrm{~mm}$.

IMAGE ANALYSIS

The CT images were enlarged, visualised on a computer screen, and analysed. Analysis of the multifidus muscle consisted of two steps using a computer program.

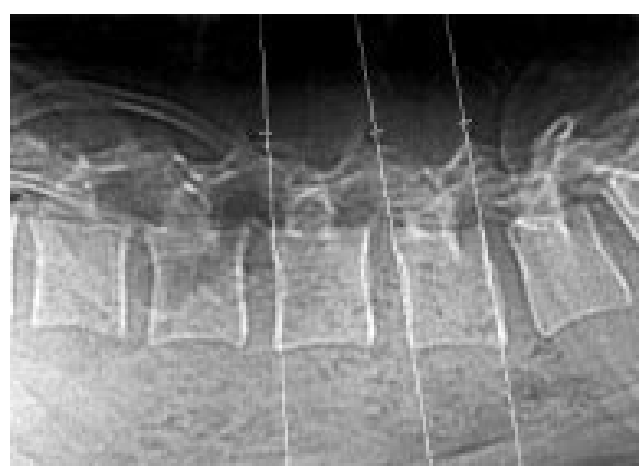

Figure 1 A scouth view representing the three standardised views positioned accurately along the upper end plate of L3 and the upper and lower end plate of L4.

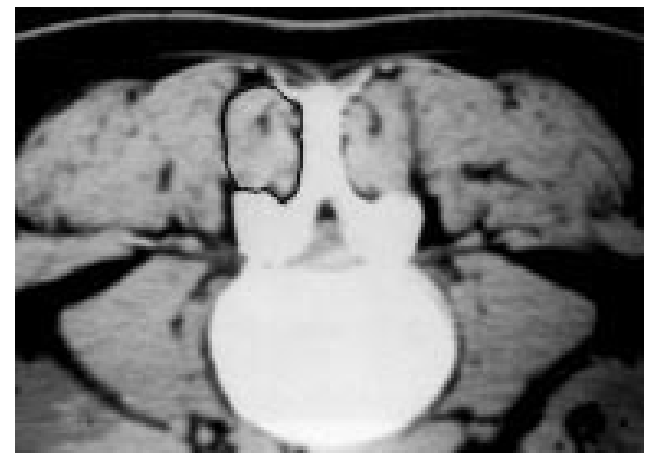

Figure 2 The cross sectional area of the multifidus muscle was measured on the transaxial view. The outlines of the region of interest were identified by cursor on the computer screen.

To determine the region of interest, the margins of the muscle were identified by cursor on the computer screen. This CSA was considered to represent multifidus muscle with fat (fig 2).

The next CSA was exposed by an elimination technique. Image segmentation was performed using a threshold technique based on the differences in the grey values of the pixels. Bone and clear fat deposits were eliminated. These eliminations resulted in the second CSA: "the low fat multifidus tissue" (fig 3). Only the low fat multifidus tissue was used to evaluate the effects of the treatment.

This measurement technique has been shown to be reliable, ${ }^{13}$ and all measurements were made by one observer who was blinded to all subject information to eliminate potential bias.

\section{DATA ANALYSIS}

To assess changes within each group after the intervention period, the raw data were used (amount of pixels). Both right and left sides were studied, and the arithmetic sums of the right and left multifidus were calculated. ${ }^{13} 172232$

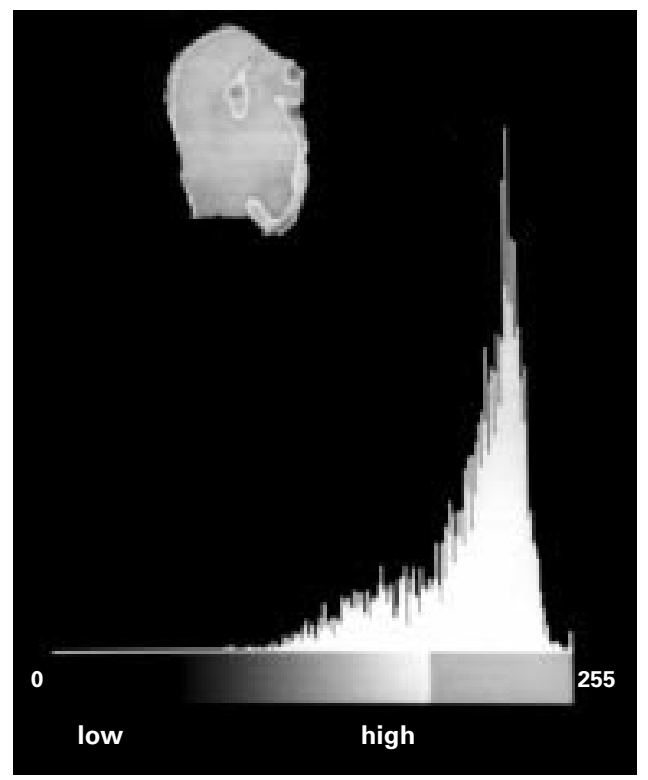

Figure 3 Histographic method used to isolate muscle tissue from fat deposits. 
Table 2 Cross sectional areas of the multifidus muscle before and after the three different training modalities

\begin{tabular}{llll}
\hline & \multicolumn{1}{l}{ Before } & After & p Value \\
\hline Level I & $10312.95(2173.37)$ & $10351.11(2535.45)$ & 0.97 \\
Group 1 & $10810.14(2226.41)$ & $10847.95(2346.19)$ & 0.79 \\
Group 2 & $9970.63(2878.94)$ & $10613.95(3226.04)$ & 0.014 \\
Group 3 & & & \\
Level II & $14956.90(2505.43)$ & $14929.58(2693.19)$ & 0.49 \\
Group 1 & $15541.37(3460.09)$ & $15781.04(3363.08)$ & 0.16 \\
Group 2 & $14933.35(3570.45)$ & $15871.58(4350.62)$ & 0.008 \\
Group 3 & $20474.21(2854.57)$ & $20419.53(2961.50)$ & 0.809 \\
Level III & $20944.07(3382.33)$ & $21341.40(3499.22)$ & 0.25 \\
Group 1 & $19878.27(4241.25)$ & $21310.58(4910.20)$ & 0.002 \\
Group 2 & & & \\
Group 3 &
\end{tabular}

Results are expressed as pixels and are mean (SD). Differences produced by the treatment are given as p values. Significance level $=0.05$. Group 1, 10 weeks of stabilisation training; group 2, 10 weeks of stabilisation training combined with dynamic resistance training; group 3, 10 weeks of stabilisation training combined with dynamic-static resistance training.

To assess group differences at entry to the trial, the baseline data were expressed as a percentage of the CSA of the vertebral body. The relative values were necessary to compare the three different groups. ${ }^{16} 32$

STATISTICAL ANALYSIS

The data are presented as mean (SD). A repeated measure analysis of variance was applied in an exploratory fashion. However, as the data showed no normal distribution (Kolmogorov-Smirnov test significant), parametric tests could not be used. Therefore nonparametric alternatives were applied. Within all groups, treatment effects were analysed with the Wilcoxon test. On the relative baseline data, a Kruskall-Wallis test was performed to assess group differences at entry to the trial. For statistical analysis, the software SPSS 9.0. was used. Statistical significance was accepted at the $5 \%$ level.

\section{Results}

Non-parametric statistical analysis showed no significant differences between the groups on entry to the trial (table 1). Analysis of differences within each group after the intervention period showed significant differences in group 3 at the three levels $(\mathrm{p}=0.014,0.008$, and 0.002 for levels I, II, and III respectively). In groups 1 and 2 , no significant differences were found (table 2).

Stabilisation training (group 1) and stabilisation training combined with progressive dynamic resistance (group 2) have no significant effect on the size of the multifidus muscle. On the other hand, stabilisation training combined with progressive dynamic-static resistance training (group 3) has.

\section{Discussion}

In this study no other effect parameters such as muscle strength or muscle coordination were measured. Although dynamometry and maximal effort may be problematic in patients, these parameters may have added valuable information. Despite this limitation, the data on the CSA of the multifidus muscle provide clinically relevant information.

To determine which results can be solely attributed to the progressive resistance training, in group 1, the treatment consisted of diathermy, general stabilisation exercises, and massage only. As this training was found to have no effect on the CSA, the results support the idea that, to increase muscle mass, an intensive strengthening programme is necessary in addition to stabilisation training.

Although several studies have reported positive effects of stabilisation training on neuromuscular function and muscle condition in patients with CLBP, ${ }^{23}$ to our knowledge only one study has used the CSA to evaluate stabilisation training. Hides et $a l^{34}$ found that localised physical training restored the size of the multifidus muscle in patients with acute low back pain. The results of our study suggest that, in patients with CLBP, generalised stabilisation training is not sufficient to increase the CSA of the back muscles. However, the comparison of the two studies has several limitations that should be considered. Firstly, in contrast with our more generalised stabilisation training, Hides et al focused on non-resisted isometric co-contraction of multifidus and deep abdominal muscles. Moreover, the occurrence of a multifidus contraction was verified in the treatment session using feedback from real-time ultrasound imaging.

Secondly, it may be that the internal structural changes in the multifidus muscle are different in the acute (used in the study of Hides et al) and chronic (used in this study) stage of low back pain. The results of Ford et $a l^{35}$ indicate that these changes are present in type I fibres in patients who have experienced pain for only three weeks, whereas several other authors have found that mainly the size of type II fibres is reduced in patients with chronic back pain. ${ }^{36}{ }^{37}$ Thirdly, in the chronic stage, recovery of the multifidus muscle may be hampered by changed recruitment patterns, so that other muscles are active and substitute for the stabilising muscles, particularly the multifidus. ${ }^{9}$ Possibly, generalised stabilisation training is not specific enough to alter these recruitment patterns.

In groups 2 and 3, a progressive resistance training mode was used. The physiological adaptations most often found after high resistance training include increases in muscle mass and strength, in bone mass, and in connective tissue thickness. ${ }^{38}$

The dynamic training mode caused no significant increase in the CSA of the multifidus. In contrast, other studies have shown significant increases in the CSA after dynamic extension exercises. ${ }^{24}{ }^{39}$ Although an exact comparison between the different programmes is not possible, the observed differences may be explained by differences in the intensity of the rehabilitation programmes and the fact that different back muscles are analysed. In the study of Foster et $a l,{ }^{39}$ the CSA of the erector spinae were evaluated before and after dynamic training at $80 \%$ of $1 \mathrm{RM}$. The CSA of the multifidus was not taken into account. In the study of Parkkola et al, ${ }^{24}$ the training included various types of submaximal (40-60\%) muscle training modalities and, as the erector spinae were evaluated together with the multifidus, we have no idea of the CSA of the isolated multifidus. 
Skeletal muscles consist of two main fibre types. These are divided according to their contractile properties into slow twitch fibres, with a large potential for endurance, and fast twitch fibres, with a capacity for forceful contraction. Muscle biopsy specimens from patients having a discectomy have shown selective atrophy of type II fibres in low back muscles. ${ }^{363740}$ However, selective type II fibre atrophy has also been encountered in people with no known back problems. ${ }^{3641}$ The authors suggested that people today with sedentary lifestyles do not expose their back muscles to work loads high enough to stimulate the type II fibres and retain their normal size. This view was further supported by a follow up study in which normalisation of type II fibre size was clearly found in patients who had returned to normal life after a successful disc operation. ${ }^{37}$

In stabilisation training consisting of repetitive low intensity exercises and postural demands, the fast twitch fibres may hardly ever be recruited. ${ }^{42}$ In dynamic exercises at $70 \%$ of the $1 \mathrm{RM}$, hypertrophy of the type II fibres was expected. Rissanen $e t a l^{43}$ found that fast twitch fibres of the multifidus recover as a result of intensive exercise. They showed that training with maximal and submaximal effort may reverse the selective atrophy of type II fibres in the multifidus. Submaximal effort consisted of loads of $80 \%$ of the $1 \mathrm{RM}$ three days a week. Maximal strength exercises were carried out twice a week using loads of $90-100 \%$ of the 1 RM. A possible explanation for the differences from our findings is that maximal demands are required to recruit the fast twitch fibres during dynamic exercise.

In contrast with the findings of Rissanen et $a l,{ }^{4342}$ the results of Goldspink ${ }^{42}$ indicate that the fast contracting fibres are recruited only in rapid power movements or high intensity isometric contractions. This selective response depending on the type of exercise may clarify the differences found between dynamic and dynamic-static training. As rapid power movements or high intensity isometric contractions are necessary to recruit fast twitch fibres, the static holding component at $70 \%$ of $1 \mathrm{RM}$ may have been the critical stimulus.

To date, information on the relative activity of the erector spinae and the multifidus in dynamic and combined dynamic-static contraction modalities is lacking. Moreover, the influence of different types of muscle work on the CSA of the back muscles has not been evaluated. In the lower extremities, studies have identified different muscular responses to concentric and eccentric work, indicating that muscle adaptive responses vary in function with the specific training regimen used. ${ }^{28}{ }^{29}$ The hypertrophic response appeared to be more prominent when eccentric muscle actions were performed. In our study, the eccentric workload was matched over both groups who followed an intensive strengthening programme. The results indicate that the static holding component between the concentric and eccentric phase is critical in inducing muscle hypertrophy during the first 10 weeks. The dynamic-static training mode has been recommended in order to recruit as many motor units as possible. ${ }^{30}$ The results indicate that the physiological adaptations of the multifidus muscle to pure dynamic and combined staticdynamic muscle activity are different, to such an extent that a systematic difference in CSA could be found. According to Welsh and Rutherford, ${ }^{44}$ in exercises incorporating an isometric phase, a greater level of metabolite accumulation can be expected for two reasons. Firstly, the duration of these exercises is much longer than in the dynamic mode, and, secondly, the static component causes higher intramuscular pressure, reducing oxygenation in a significant way. ${ }^{45}$

Controversy exists about the metabolic stimulus for muscle hypertrophy. Contractions of high force produce high mechanical stress in the muscle at great metabolic cost. Conventionally, the former has been considered to be the critical stimulus, but there is evidence that the metabolic cost of exercise is also important. ${ }^{44}$ The findings that, in contrast with the dynamic training, which had no hypertrophic effect, the controlled application of combined dynamic-static overload seems to be necessary to obtain a volume increasing effect could support the importance of metabolic factors.

\section{CONCLUSION}

The results of this study suggest that general stabilisation exercises and dynamic intensive lumbar resistance training have no significant effect on the CSA of the lumbar multifidus muscle in patients with CLBP. The static holding component between the concentric and eccentric phase was found to be critical in inducing muscle hypertrophy during the first 10 weeks.

A treatment routine consisting of stabilisation training combined with dynamic-static workload for the paravertebral muscles seems to be the most appropriate method for reversing atrophy of the multifidus muscle.

We thank Ms Hilda Raes and Mr Robin Hellebuyck for their assistance in collecting the data.

1 Andersson G. The epidemiology if spinal disorders. In: Frymoyer JW, ed. The adult spine: principles and practice. New York: Ravel Press, 1991:107-46.

2 Johannsen F, Remvig L, Kryger P, et al. Exercises for chronic ow back pain: a clinical trial. F Orthop Sports Phys Ther 1995;22:52-8.

3 Mayer T, Vanharanta H, Gatchel R, et al. Comparison of CT scan muscle measurements and isokinetic trunk strength in postoperative patients. Spine 1989;14:33-6.

4 Waddell G. A new clinical model for the treatment of low-back pain. Spine 1987;12:632-44.

5 Pollock M, Leggett S, Graves J, et al. Effect of resistance Pollock M, Leggett S, Graves J, et al. Effect of resistance
training on lumbar extension strength. Am f Sports Med training on lum

6 Friberg O. Lumbar instability: a dynamic approach by traction-compression radiography. Spine 1987;12:119-29. 7 Nachemson A. Instability of the lumbar spine. Neurosurg Clin N Am 1991;2:785-90.

8 Panjabi M. The stabilizing system of the spine. Part I Function, dysfunction, adaptation, and enhancement. I Spinal Disord 1992;5:383-9.

9 O' Sullivan P, Phyty G, Twomey L, et al. Evaluation of specific stabilizing exercise in the treatment of chronic low back pain with radiologic diagnosis of spondylolysis or spondylolisthesis. Spine 1997;22:2959-67.

10 Danneels LA, Vanderstraeten GG, Cambier DC, et al. A functional subdivision of hip, abdominal and back muscles during asymmetric lifting. Spine 2001;26:E114-21.

11 Goel V, Kong W, Han J, et al. A combined finite element and optimization investigation of lumbar spine mechanics with and without muscles. Spine 1993;18:1531-41. 
12 Wilke $\mathrm{H}$, Wolf S, Claes L, et al. Stability increase of the lumbar spine with different muscle groups. A biomechanical in bar spine with different muscle grou
vitro study. Spine 1995;20:192-8.

13 Danneels L, Vanderstraeten G, Cambier D, et al. SSE Clinical Science Award 2000: computed tomography imaging of trunk muscles in chronic low back pain patients and healthy control subjects. Eur Spine f 2000;9:266-72.

14 Kader D, Wardlaw D, Smith F. Correlation between the MRI changes in the lumbar multifidus muscles and leg pain. Clin Radiol 2000;55:145-9.

15 Hides J, Stokes M, Saide M, et al. Evidence of lumbar multifidus muscle wasting ipsilateral to symptoms in patients with acute/subacute low back pain. Spine 1994;19:165-72

16 Cooper R, Clair Forbes W, Jayson M. Radiographic demonstration of paraspinal muscle wasting in patients with
chronic low back pain. Br f Rheumatol 1992;31:389-94.

17 Hultman G, Nordin M, Saraste H, et al. Body composition, endurance, strength, cross-sectional area, and density of $\mathrm{mm}$ erector spinae in men with and without low back pain. m Spinal Disord 1993;6:114-23.

18 Keller A, Johansen J, Hellesnes J, et al. Predictors of isokinetic back muscle strength in patients with low back pain. Spine 1999;24:275-80.

19 Sihvonen T, Herno A, Paljärvi L, et al. Local denervation atrophy of paraspinal muscles in postoperative failed back syndrom. Spine 1993;18:575-81.

20 Flicker P, Fleckenstein J, Ferry K, et al. Lumbar muscle usage in chronic low back pain. Magnetic resonance image evaluation. Spine 1993;18:582-6.

21 Gibbons L, Videman T, Battié M. Isokinetic and psychophysical lifting strength, static back muscle endurance, and magnetic resonance imaging of the paraspinal muscles as predictors of low back pain in men. Scand 7 Rehabil Med 1997;29:187-91.

22 Parkkola R, Rytökoski U, Kormano M. Magnetic resonance imaging of the discs and trunk muscles in patients with chronic low back pain and healthy control subjects. Spine 1993;18:830-6.

23 Peltonen J, Taimela S, Erkintalo M, et al. Back extensor and psoas muscle cross-sectional area, prior physical training, psoas muscle cross-sectional area, prior physical training, and trunk muscle strength: a longitudinal stud

24 Parkkola R, Kujala U, Rytökoski U. Response of the trunk muscles to training assessed by magnetic resonance imaging and muscle strength. Eur f Appl Physiol 1992;65 383-7.

25 Carpenter D, Nelson B. Low back strengthening for the prevention and treatment of low back pain. Med Sci Sports Exerc 1999;31:18-24

26 Jull G, Richardson C. Rehabilitation of active stabilisation of the lumbar spine. In: Twomey LT, Taylor JR, eds. Physical therapy of the low back. 2nd ed. New York: ChurchillLivingstone, 1994:251-73.

27 Manniche C, Hesselфe G, Bentzen L, et al. Clinical trial of intensive muscle training for low chronic low back pain. Lancet 1988;31:1473-6.

28 Hather B, Tesch P, Buchanan P, et al. Influence of eccentric actions on sceletal muscle adaptations to resistance training. Acta Physiol Scand 1991;143:177-85.
29 Walker P, Brunotte F, Rouhier-Marcer I, et al. Nucleair magnetic resonance evidence of different muscular adaptations after resistance training. Arch Phys Med Rehabil 1998; 79:1391-8.

30 Swinkels J. [The logic of sports training and important tendencies.] (In Dutch) De Richting 1981;35:150.

31 O'Sullivan P, Twomey L, Allison G. Dynamic stabilization of the lumbar spine. Critical Reviews Physical Rehabilitation Medicine 1997;9:315-30.

32 McLoughin R, D’Arcy E, Brittain M, et al. The significance of fat and muscle areas in the lumbar paraspinal space: a CT study. $\mathcal{F}$ Comput Assist Tomogr 1994;18:275-8.

33 Han J, Ahn J, Goel V, et al. CT-based geometric data of human spine musculature. Part I. Japanese patients with chronic low back pain. 7 Spinal Disord 1992;5:448-58.

34 Hides J, Richardson C, Jull G. Multifidus recovery is not automatic following resolution of acute first episode of low back pain. Spine 1996;21:2763-9.

35 Ford D, Bagnall K, McFadden $\mathrm{H}$, et al. Analysis of vertebral muscle obtained during surgery for correction of a lumbar disc disorder. Acta Anat 1983;116:152-7.

36 Mattila $M$, Hurme $M$, Alaranta $H$, et al. The multifidus muscle in patients with lumbar disc herniation. A histochemical and morphometric analysis of intraoperative biopsies. Spine 1986;11:732-8.

37 Rantanen J, Hurme M, Falck B, et al. The lumbar multifidus muscle five years after surgery for a lumbar intervertebral disc herniation. Spine 1993;18:568-74.

38 Feigenbaum M, Pollock $M$. Strength training: rationale for current guidelines for adult fitness programs. Physician and Sportsmedicine 1997;25:44-64.

39 Foster D, Avillar M, Pollock M, et al. Adaptations in strength and cross-sectional area of the lumbar extensor muscles following resistance training. Med Sci Sports Exerc 1993;25(suppl):47.

40 Zhu X-Z, Parnianpour M, Nordin M, et al. Histochemistry and morphology of erector spinae muscle in lumbar disc herniation. Spine 1989;14:391-7.

41 Rantanen J, Rissanen A, Kalimo H. Lumbar muscle fiber size and type distribution in normal subjects. Eur Spine $\mathcal{F}$ 1994;3:331-5

42 Goldspink G. Cellular and molecular aspects of adaptation in skeletal muscle. In: Komi PV, ed. Strength and power in sport. Oxford: Blackwell Scientific Publications, 1992;21129.

43 Rissanen A, Kalimo H, Alaranta H. Effect of intensive training on the isokinetic strength and structure of lumbar muscles in patients with chronic low back pain. Spine 1995;20: $333-40$.

44 Welsh L, Rutherford O. Effects of isometric strength training on quadriceps muscle properties in over 55 year olds. Eur F Appl Physiol 1996;72:219-23.

45 Jensen B, Jorgensen K, Hargens A, et al. Physiological response to submaximal isometric contractions of the paravertebral muscles. Spine 1999;24:2332-8.

\section{Take home message}

CT examination found selective atrophy of the multifidus muscle in patients with CLBP. This may be the cause of spinal instability and contribute to the high recurrence rate in CLBP. Treatment consisting of stabilisation training combined with dynamic-static workload for the paravertebral muscles would seem to be an appropriate method for reversing this atrophy. 